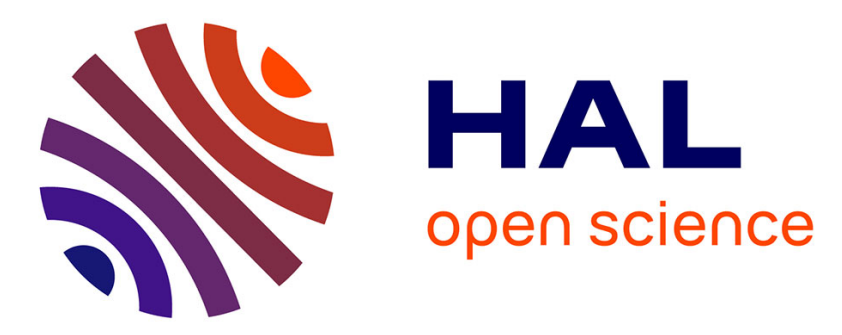

\title{
Premiers Pointages chez L'enfant entendant et L'enfant sourd-signeur: deux suivis longitudinaux entre 7 mois et 1 an 7 mois
}

\author{
Aliyah Morgenstern, Emmanuelle Mathiot, Marie Leroy, Fanny Limousin
}

\section{- To cite this version:}

Aliyah Morgenstern, Emmanuelle Mathiot, Marie Leroy, Fanny Limousin. Premiers Pointages chez L'enfant entendant et L'enfant sourd-signeur: deux suivis longitudinaux entre 7 mois et 1 an 7 mois. AILE - LIA, 2009, 1 (1), pp.141-168. 10.4000/aile.4515 . halshs-00529889

\section{HAL Id: halshs-00529889 \\ https://shs.hal.science/halshs-00529889}

Submitted on 26 Oct 2010

HAL is a multi-disciplinary open access archive for the deposit and dissemination of scientific research documents, whether they are published or not. The documents may come from teaching and research institutions in France or abroad, or from public or private research centers.
L'archive ouverte pluridisciplinaire HAL, est destinée au dépôt et à la diffusion de documents scientifiques de niveau recherche, publiés ou non, émanant des établissements d'enseignement et de recherche français ou étrangers, des laboratoires publics ou privés. 


\title{
PREMIERS POINTAGES CHEZ L'ENFANT ENTENDANT ET L'ENFANT SOURD-SIGNEUR : deux suivis longitudinaux entre 7 mois et 1 an 7 mois
}

\author{
Emmanuelle MATHIOT, Marie LEROY, \\ Fanny LIMOUSIN, Aliyah MORGENSTERN ${ }^{1}$ \\ (Université Lille 3 \& STL ; Université Paris Descartes \& Modyco ; \\ Université Paris 8 \& STL ; Université Sorbonne Nouvelle Paris 3 \& ICAR)
}

\begin{abstract}
RÉSUMÉ
Le pointage émerge grâce à la maîtrise des pré-requis moteurs et cognitifs et facilite les processus de symbolisation. Ce geste représente une condition nécessaire à la construction du langage car il donne à l'enfant la possibilité de désigner un objet en tant que lieu d'attention partagée et d'échange avec l'adulte. Par la comparaison entre les suivis longitudinaux de deux petites filles, dont l'une est entendante et élevée en milieu francophone et l'autre sourde et élevée dans un environnement signeur, nous essaierons de déterminer si les éléments qui accompagnent les gestes de pointage (regards, vocalisations) et les paramètres qui caractérisent «l'événement pointage » (situation de la cible du pointage, situation de l'interlocuteur, statut de l'objet dans le discours et attention partagée ou non) permettent de distinguer les fonctions proto-déclarative et proto-impérative décrites dans la littérature.

(Mots-clés : pointage, enfant sourd-signeur, multimodalité, regard, prosodie)
\end{abstract}

1. Adresse des auteurs : emmanuelle.mathiot@univ-lille3.fr, Université Lille 3, CNRSUMR 8163 STL; marie.leroy@parisdescartes.fr, Université Paris Descartes, CNRSUMR 7114 MODYCO ; fannylimou@gmail.com, Université Paris 8, CNRS-UMR 7023 SFL ; aliyah.morgenstern@univ-paris3.fr, Université Sorbonne NouvelleParis 3, CNRS-UMR 5191 ICAR.

Aile - LIA 1 - 2009 


\section{Introduction}

\subsection{Spécificité du pointage}

Le geste de pointage fait partie des formes gestuelles conventionnelles comprises et produites par les enfants, dès la fin de la première année (Bates 1976; Bates et al. 1979; Guidetti 1998), qu'ils soient sourds ou entendants. Il manifeste des compétences socio-cognitives qui sont la base des interactions langagières (Tomasello 1999, 2003). Il s'agit en effet tout d'abord d'un geste social, qui permet d'établir l'attention conjointe avec un partenaire (Schaffer 1977, 1984). C'est également un geste référentiel, puisqu'il isole dans l'environnement un objet d'intérêt vers lequel cette attention est dirigée (Bruner 1977, 1983). Comme le soulignent Moore et Dunham (1995), un triangle référentiel est ainsi mis en place. Il permet la communication intersubjective préalable aux échanges linguistiques (oraux ou signés) qui apparaissent généralement au même âge ou peu après. Par les opérations cognitives et psychiques qu'il implique, ce serait un geste propre aux interactions humaines (Butterworth 2003; Pika et al. 2005; Tomasello et Camaioni 1997), et l'un des premiers éléments observables du comportement communicatif humain, indiquant que l'enfant, contrairement à l'animal, est capable de construire l'altérité psychique.

\subsection{Différences entre les modalités audio-orale et visuo-manuelle}

Cette étude étant basée sur des données longitudinales d'une enfant sourde de parents sourds-signeurs et d'une enfant entendante de parents entendants, il convient tout d'abord de rappeler que des distinctions importantes existent entre les interactions dans les deux situations. Tout d'abord, l'articulation des activités quotidiennes et des échanges langagiers est différente. Dans une dyade entendante, la mère s'adonne souvent à d'autres activités tout en parlant à son enfant. Dans une dyade sourde, il est pratiquement impossible à la mère de communiquer par signes avec son enfant tout en ayant une autre activité manuelle : elle ne peut à la fois signer et faire la cuisine, changer la couche, jouer... Par ailleurs, elle est constamment dans « l'écoute visuelle» de son enfant. On a donc un mode interactionnel très spécifique dans lequel les yeux remplacent les oreilles chez les deux partenaires. Dans les débuts de l'acquisition du langage chez les enfants sourds-signeurs, on constate d'ailleurs que leurs parents sourds travaillent à la mise en place de cette concentration visuelle, ce que Morgan (2009) appelle « learning to look ». Les mères étudiées par Van den Bogaerde (2000) s'assurent ainsi avant de signer que leur enfant peut voir leurs mains et 
leur visage. Pour pouvoir communiquer par signes, il est nécessaire d'attirer l'attention visuelle de l'interlocuteur, puis de la maintenir constamment. On ne peut donc pas « écouter » si on ne regarde pas l'autre, contrairement à ce qui se passe entre entendants, où le regard n'est pas absolument indispensable. Très tôt d'ailleurs, dans nos données, l'enfant sourde semble comprendre que pour couper court au dialogue, il suffit de couper le contact visuel. Ainsi, à 10 mois, elle tourne délibérément le dos à sa mère lorsque celle-ci lui interdit de mettre des jouets dans sa bouche.

Il en résulte qu'en langue des signes, le langage adressé à l'enfant n'est jamais ou presque parasité par d'autres activités. Les moments de langage vont être intenses, concentrés. Le langage est une « mono-activité ». Et inversement, les autres activités s'accompagnent rarement de langage, ou pas de manière continue : la mère ne commente pas en permanence ce qu'elle fait comme pourrait le faire une mère entendante d'enfant entendant.

Par ailleurs, dans la langue des signes, le geste et le regard priment, alors que dans la langue orale, c'est davantage la modalité audio-orale, même si le visuo-manuel reste utilisé. Un enfant exposé à la langue des signes est donc plus sensible aux gestes, et les adultes sourds vont très tôt interpréter les ébauches gestuelles de leurs enfants, tout comme les adultes entendants le font avec les vocalisations des enfants entendants. Or les gestes semblent plus accessibles, plus « faciles » à produire que les mots au niveau neuro-psychomoteur. On s'aperçoit que les enfants exposés à une langue des signes font des gestes beaucoup plus précis de façon plus précoce que les enfants exposés uniquement à une langue orale (alors qu'ils ont a priori les mêmes bagages psycho-moteurs). La configuration « index levé, autres doigts repliés » est une des premières et des plus fréquentes configurations dans les premiers signes des enfants sourds-signeurs (Engberg-Petersen 1993a, b; Boyes Braem 1994; Conlin et al. 2000). Les enfants sourds signeurs explorent les possibilités offertes dans la modalité gestuelle pendant une période plus longue que les enfants entendants. Par ailleurs, on peut aider un enfant à faire un geste, le lui corriger en lui manipulant les mains, mais on ne peut pas agir directement et physiquement sur son appareil phonatoire.

\subsection{Pourquoi comparer enfants sourds et enfants entendants}

Le pointage prendra un statut différent selon la modalité de communication employée. Au moment de son apparition, le pointage est un geste de désignation (d'un lieu, d'un objet, d'une personne, d'un événement) aussi bien chez l'enfant entendant que chez l'enfant sourd-signeur. Mais pour l'enfant signeur, ce geste va s'intégrer au système formel de la langue (ici, la Langue des 
Signes Française, ou LSF) et avoir le même statut que des marqueurs comme les pronoms personnels et démonstratifs en français, tout en restant occasionnellement utilisé comme geste non-linguistique. Chez l'enfant entendant par contre, le pointage reste un geste de désignation, d'abord isolé puis complémentaire du verbal. L'analyse du pointage en langue des signes constitue donc une occasion unique d'observer l'éventuelle continuité ou discontinuité entre gestes et signes (dans le sens saussurien du terme) et de nous éclairer sur le fonctionnement des déictiques qui semblent prendre le relais des gestes de pointage chez l'enfant entendant. Notre étude ne sera pas consacrée à cette question qu'il nous sera possible d'aborder dans un prolongement de ce travail, mais il nous paraît important de comparer les pointages dès leur émergence et dans la période pré-linguistique avant d'aborder une période plus tardive. Or, si des travaux comme ceux de Caselli et Volterra (1994) comparent les premiers gestes, signes et mots d'enfants sourds et enfants entendants en milieu naturel, il existe peu d'études spécifiquement consacrées aux pointages, ce que nous nous proposons de faire ici.

Nous ferons d'abord une revue des principales problématiques abordées dans la littérature qui ont servi de point de départ à notre travail. Nous présenterons ensuite notre méthodologie de travail sur les pointages de Madeleine, petite fille entendante, et de Charlotte, petite fille sourde et nos analyses quantitatives et qualitatives. Notre objectif est de comparer les spécificités des « évènements-pointage » que constituent l'ensemble formé par le pointage, le regard, la prosodie de la voix quand il y a émission vocale, en les replaçant dans leur contexte et en fonction des objets désignés.

\section{Pointage et entrée dans la langue}

\subsection{Origines du pointage}

Le pointage permet d'établir un lien entre soi et l'objet pointé. On a pu considérer que la forme gestuelle du pointage proviendrait d'un mouvement non abouti pour saisir un objet (Wundt 1912), ou du comportement d'exploration de l'index déjà présent à 3 mois, qui avec l'extension du bras serait ensuite étendu aux objets inaccessibles par le toucher (Masataka 2003).

Il en découle deux interprétations possibles de la mise en place des usages que l'enfant en a (Marcos 1998). Dans la lignée de Vygotsky (1985), le pointage naîtrait de l'interaction sociale entre adulte et enfant; le geste initial de la main pour saisir un objet hors d'atteinte serait interprété par l'adulte comme expression du désir de prendre cet objet, d'où en retour une utilisation par 
l'enfant du geste d'index pointé pour diriger l'attention de l'adulte vers un objet convoité afin d'obtenir ce dernier. Une autre explication généralement rattachée à Werner et Kaplan (1963) serait que l'enfant utilise d'abord le pointage pour lui-même, et isolerait ainsi par le geste un objet digne d'intérêt, ce qui pose les bases primitives d'une activité référentielle.

On peut également considérer que le geste de pointage est tout simplement appris par imitation des interlocuteurs adultes qui utilisent eux-mêmes le pointage pour attirer l'attention de l'enfant. (Leung et Rheingold 1981; Kaye 1982).

\subsection{Usages et fonctions du pointage}

Le pointage est l'un des gestes les plus étudiés dans le développement de la communication et du langage, notamment depuis les travaux de Bates (1976) et Bates et al. (1979) sur les compétences pragmatiques précoces. Selon ces auteurs, on peut distinguer deux valeurs du pointage, selon qu'il sert à obtenir (pointage proto-impératif) ou à référer (pointage proto-déclaratif). Si la plupart des travaux sur les débuts du langage chez l'enfant ont repris cette distinction, des études expérimentales récentes l'ont enrichie en suggérant d'autres fonctions du pointage. Liszkowski et al. (2006) ont ainsi mis en évidence un usage informatif du pointage chez les bébés de 12 mois, qui seraient capables de l'utiliser pour transmettre à autrui un savoir connu d'eux seuls (en l'occurrence, la localisation d'un objet égaré par l'adulte). L'enfant peut également de façon très précoce utiliser le geste de pointage pour obtenir de l'adulte des informations sur le monde environnant, ce qui en fait un mécanisme d'apprentissage culturel et linguistique (Southgate et al. 2007).

\subsection{Pointage et deixis}

Chez tous les enfants, qu'ils soient sourds ou entendants, qu'ils grandissent dans un environnement qui signe ou qui parle, la capacité à comprendre la valeur de deixis manifestée par le geste de pointage à la fin de la première année (Cabrejo-Parra 1992), et à produire à leur tour ce geste avec l'intention de faire référence indique une aptitude essentielle pour la mise en place du langage, la compréhension du fait que les objets ont un nom. Ainsi, Harris et al. (1995) ont mis en évidence une relation entre l'âge des premiers pointages et l'âge où les premiers noms d'objet sont compris par les enfants. L'adulte pointe pour l'enfant, et peu de temps après l'enfant pointe pour l'adulte, vers un objet d'attention conjointe qui devient alors un référent. Ninio et Bruner (1978) 
ont montré qu'isoler ainsi un objet par le geste de pointage crée des conditions favorables aux activités de labeling.

De ce fait, les travaux qui portent sur le développement du langage oral voient généralement dans le pointage un acte précurseur de la dénomination. Cependant, certaines études sur le développement des langues signées (Bonvillian et al. 1994) soulignent que dans cette modalité, le pointage ne précède pas le langage, parce que chez les enfants signeurs observés, pointage et premiers signes apparaissent conjointement, et plus tôt que chez les enfants qui acquièrent une langue orale. Ces auteurs considèrent donc que le pointage n'est pas un pré-requis au langage, contrairement à ce qu'ont conclu de nombreux travaux sur l'acquisition des langues orales.

\section{4. Âge d'apparition du pointage : comparaison entre langues orales et langues signées}

Le pointage apparaît donc plus tôt chez les enfants dont l'entourage signe que chez ceux dont l'entourage communique en langue orale, de même d'ailleurs que les premiers signes semblent apparaître plus tôt que les premiers mots. La motricité fine de l'appareil phonatoire que nécessite la production du langage oral est en effet maîtrisée plus tardivement que celle des mains, qui sont les articulateurs de la langue des signes. La production de mots est donc limitée par la maîtrise incomplète de l'appareil phonatoire, sur lequel aucun contrôle visuel n'est possible, alors même que les enfants seraient cognitivement prêts à utiliser des unités lexicales comme en atteste la production de signes manuels avant la fin de la première année (Emmorey 2002; Schick 2007).

Par ailleurs, peu de travaux portent sur la compréhension du pointage par les enfants. A partir de quel âge, en fonction de quels acquis socio-cognitifs, un enfant considère-t-il le pointage de l'adulte comme un geste de communication qui n'a pas d'intérêt ni de contenu de sens en lui-même, mais lui indique un objet digne d'intérêt ? Selon Murphy et Messer (1977) dont l'étude porte sur des dyades mère-enfant entendantes, certaines conditions (liées à la position de l'objet par rapport aux partenaires de l'interaction) peuvent être réunies dès 9 mois, ce qui indique déjà une certaine conscience de la valeur du geste de l'interlocuteur. Mais ce n'est qu'à 14 mois que les bébés suivent régulièrement du regard la direction des pointages adultes quelle que soit leur orientation. Etant donné que dans ces dyades, adulte et enfant communiquent aussi par la modalité verbale, on peut se demander si dans des dyades où la modalité principale est visuo-gestuelle, parce que l'adulte s'exprime uniquement en langue des signes, l'enfant ne prêterait pas une attention plus précoce aux pointages maternels, 
simplement parce qu'il est particulièrement attentif aux manifestations gestuelles de l'adulte en général.

\subsection{Le rôle du regard}

Les travaux antérieurs sur le pointage soulignent généralement l'importance du regard et, pour les enfants entendants, des vocalisations accompagnant ce geste. Ces deux éléments manifestent la valeur communicative du geste de pointage (Franco et Butterworth 1996). En effet, le regard est considéré comme l'indice d'une intentionnalité, notamment parce qu'il est utilisé par l'enfant dès 12-15 mois pour s'assurer que l'attention conjointe est établie avec le partenaire. La combinaison [pointage + regard] est également considérée comme un facteur prédictif du développement ultérieur du langage, que ce soit dans les modalités orale ou signée. Elle serait en effet corrélée au développement lexical précoce chez l'enfant entendant pour Bates et al. (1979), et au développement du lexique parlé et signé dans la deuxième année chez des enfants entendants et sourds pour Folven et al. (1984/85). Quant aux vocalisations de différents types chez les enfants entendants, elles accompagnent le pointage dès son apparition.

\subsection{Pointages et vocalisations/verbalisations}

Une différence importante entre langue orale et langue signée est justement que la première permet d'associer les deux modalités vocale et gestuelle. Relativement peu d'études sur les gestes des enfants entendants s'intéressent aux productions orales (cris ou gémissements, vocalisations, puis verbalisations) associées aux gestes de pointage ( $c f$. cependant Lock et al. 1990). Il s'agit là d'un autre moyen que le regard pour rechercher l'attention d'un interlocuteur, qui s'affine à mesure du développement linguistique de l'enfant. En effet, si les premiers pointages apparaissent alors que l'enfant n'est pas encore entré dans le verbal, quelques mois plus tard on note que les pointages sont associés à des éléments lexicaux, puis à des verbalisations plus longues et plus complexes.

Au stade des énoncés à un terme, on peut distinguer plusieurs combinaisons, selon que le mot accompagnant le pointage est déictique (pointage $+[\mathrm{sa}]$ ), qu'il sert à nommer l'objet désigné (pointage vers la poupée + [bebe]), à le localiser (pointage + [la] / [lela]), ou qu'il s'agit d'un élément sémantique nouveau (pointage vers un objet appartenant à la mère + [mama] (Morford et GoldinMeadow 1992; Guidetti 2003). Plusieurs auteurs s'accordent sur l'hypothèse que cette dernière catégorie de combinaison semble annoncer la transition vers 
les énoncés à deux termes combinant deux informations sémantiques distinctes (Goldin-Meadow et Butcher 2003 ; Capirci et al. 1996; Volterra et al. 2005).

Dans le développement des langues signées, l'enchaînement de deux séquences gestuelles s'observe relativement tôt. Ainsi, Bonvillian et al. (1994) constatent que les enfants signeurs peuvent associer deux signes (par exemple un pointage et un autre signe) plusieurs mois avant que les combinaisons de deux termes verbaux ne soient notées chez les enfants entendants. Cependant des combinaisons de deux gestes ont également été relevées par Goldin-Meadow et Morford (1990) chez les enfants entendants, à un âge comparable à celui de leurs pairs sourds.

\section{Problématiques et méthodologie}

\subsection{Les problématiques}

Cette revue de la littérature a permis de préciser les problématiques sur lesquelles la plupart des chercheurs ont travaillé. Nous avons voulu comparer les caractéristiques, les valeurs et les fonctions du pointage associé au regard, aux productions vocales et à d'autres paramètres à partir de suivis longitudinaux d'enfants à partir de 7 mois, filmées chaque mois dans leur entourage familial. L'une (Madeleine) est entendante, son entourage parle le français oral, et l'autre (Charlotte) est sourde, d'une famille de sourds signeurs. Le regard étant généralement associé au pointage, nous avons voulu étudier les fonctions qu'il est susceptible de jouer entre enfant et adulte en milieu naturel et les différences éventuelles entre les deux modalités langagières.

Notre question de recherche porte sur la possibilité de distinguer plusieurs fonctions du pointage chez l'enfant, en particulier dans le stade pré-linguistique, et de les rattacher à certaines des fonctions des premiers mots ou énoncés. L'enfant pointerait pour obtenir un objet convoité (valeur proto-impérative), ou pour partager l'attention avec l'adulte sur un objet intéressant (valeur protodéclarative). Mais comment définir si l'enfant pointe le jouet tout en haut sur l'étagère pour que l'adulte le lui donne, ou pour montrer ce jouet nouveau qui ne se trouvait pas là avant?

Nous avons donc cherché à isoler des paramètres permettant de distinguer ces deux fonctions dans le corpus de Madeleine, la petite fille entendante vivant dans un bain linguistique francophone, et commencé à comparer nos résultats avec ceux obtenus à partir des analyses des enregistrements de Charlotte. 


\subsection{Sujets et codage des données}

Pour les deux enfants, la période étudiée va de 7 mois à 1 an et 7 mois. Elles ont été filmées en milieu naturel une heure par mois en interaction avec leur mère et d'autres membres de leur famille. Nous avons donc travaillé sur 12 heures d'enregistrements pour Madeleine et 13 heures pour Charlotte. Madeleine est une petite entendante élevée dans un milieu monolingue francophone. Charlotte est née sourde de parents sourds signeurs qui communiquent entre eux et avec elle dans la vie quotidienne en Langue des Signes Française.

Le corpus de Madeleine ${ }^{2}$ a été transcrit et aligné en utilisant le format CHAT et le logiciel CLAN (MacWhinney 2000). Tous les pointages ont été codés. Les enregistrements vidéo de Charlotte n'ont pas été transcrits mais ont été codés, en particulier les pointages, en utilisant le logiciel ELAN ${ }^{3}$ particulièrement propice à l'analyse de la modalité visuo-gestuelle. Nous avons cependant préféré établir toute notre base de données dans un premier temps à partir de l'enregistrement vidéo afin de pouvoir porter une attention spécifique aux gestes, aux mimiques, à la prosodie et de mieux prendre en compte la situation extralinguistique. Cela nous a permis de nous mettre dans une situation d'analyse comparable à celle de nos collègues qui analysent les pointages directement dans les enregistrements vidéos des enfants sourds et de pouvoir établir des comparaisons entre Madeleine et Charlotte.

Nous avons donc recensé et codé les gestes de pointage dans une base de données en prenant en compte (outre les informations liées à chaque occurrence de pointage, telles que: minutage, contexte) les paramètres suivants :

\section{1) Paramètres liés à l'enfant}

- L'âge de l'enfant ;

- Le regard de l'enfant : est-il dirigé sur l'objet pointé, sur l'adulte ou alternativement sur l'objet et l'adulte ?

- Les productions vocales/verbales ou signées de l'enfant ;

- L'intonation des productions vocales (analysée avec PRAAT ${ }^{4}$ ) : est-elle montante, descendante, plate, en cloche ou plus complexe?

2. Le corpus de Madeleine fait partie du Paris Corpus de la base de données CHILDES http://childes.psy.cmu.edu/data/Romance/French/.

3. http://www.lat-mpi.eu/tools/elan/

4. http://www.fon.hum.uva.nl/praat/ 
10 Emmanuelle Mathiot, Marie Leroy, Fanny Limousin, Aliyah Morgenstern

2) Paramètres qui dépendent de la cible du pointage

- La nature de l'objet pointé

- La situation de l'objet : est-il à portée de main de l'enfant, ou hors de portée ?

- La cible du pointage est-elle nouvelle ou ancienne?

\section{3) Paramètres qui dépendent de l'adulte}

- La situation de l'adulte par rapport à l'enfant : est-il face à l'enfant (face à face), à côté de lui (côte à côte), derrière lui, ou ailleurs ?

- L'attention de l'adulte : est-elle acquise ou non?

- L'interprétation de l'adulte interlocuteur de l'enfant (codée à partir de sa réaction) : interprète-t-il le pointage de l'enfant (et ce qui l'accompagne) comme une demande d'objet, une demande d'action, une réponse, une assertion, une dénomination... ? Parfois, l'adulte ne réagit pas aux pointages de l'enfant, ou ne les comprend pas.

\section{4) Paramètres qui dépendent de la nature de l'interaction}

- La place du pointage dans l'interaction : est-il produit à l'initiative de l'enfant, ou en réponse à une demande de l'adulte (demande de localisation d'un objet par exemple)?

\section{5) L'analyse du chercheur}

- L'interprétation du chercheur visionnant le corpus : le pointage semble-t-il plutôt impératif, plutôt déclaratif, ou est-il impossible de décider de sa valeur?

- Lorsque les productions verbales apparaissent, à quelles catégories grammaticales (au sens large) appartiennent-elles : s'agit-il de noms, de prédicats, de localisations, ou de structures complexes (nom + prédicat, nom + localisation...) ?

Les mêmes paramètres ont été utilisés pour coder les corpus de Madeleine et Charlotte. Nous présentons ici les premiers résultats de nos analyses qui concerneront le nombre de pointages par séance, le codage du regard (paramètre qui, nous l'avons expliqué, est particulièrement important dans les interactions mèreenfant, notamment lorsque la LSF est utilisée), et nos premières observations 
sur la corrélation entre les paramètres tels que nature et situation de l'objet et fonctions du pointage.

\section{Les analyses}

\subsection{Quantification du pointage}

Notre grille de codage nous a tout d'abord permis de quantifier le nombre de pointages par séance d'une heure, pour chaque enfant.

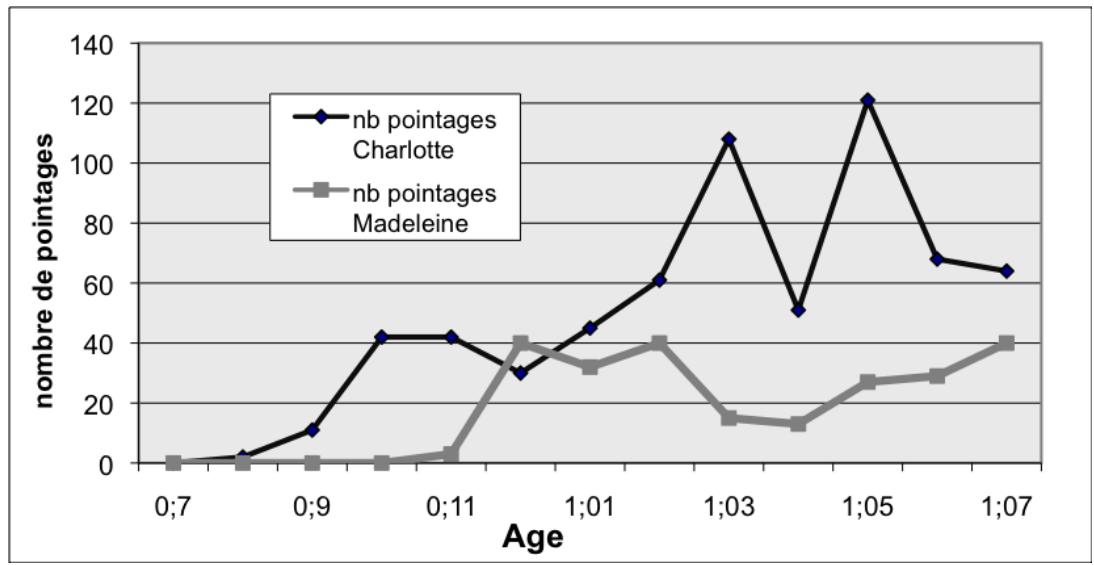

\section{Graphique 1 : Nombre de pointage s par enregistrement d'une heure dans les corpus de Madeleine et Charlotte}

Chez Madeleine, le pointage apparaît à 11 mois et augmente fortement dès 12 mois. La courbe dépend ensuite entièrement du contexte et des activités menées par la petite fille avec les adultes. Les «pics » correspondent à de longues périodes consacrées à la lecture de livres pour enfants. Au contraire, quand il y a peu d'activité de désignation, on trouve moins de pointages dans les données.

Chez Charlotte, les pointages apparaissent à 8 mois et leur nombre « explose » à partir de 10 mois. Dès cet âge, la configuration avec l'index pointé est parfaitement en place : Charlotte semble avoir une maîtrise de la motricité fine beaucoup plus précoce que Madeleine et en fait usage. Par la suite, tout comme 
chez Madeleine, le nombre d'occurrences va entièrement dépendre du contexte. Cependant, on observe que Charlotte pointe beaucoup plus que Madeleine.

\subsection{Emergence du pointage}

Grâce aux enregistrements vidéo, il nous a été possible de retracer l'histoire de l'émergence du pointage chez chacune des enfants. Le pointage semble avoir pour origine à la fois l'utilisation de l'index pour explorer (Masataka 2003) et l'utilisation de l'extension du bras pour attraper (Bates 1976). Afin d'essayer de vérifier ces origines possibles du pointage, nous avons suivi dans nos enregistrements vidéo la mise en place du pointage chez Madeleine et Charlotte.

\section{Madeleine}

A sept mois, Madeleine qui ne se déplace pas encore et ne produit pas de gestes intentionnels suit cependant du regard ce que lui montre sa mère. A neuf mois, elle a commencé à ramper, tient très bien assise et passe facilement d'une position à l'autre. Elle tend le bras, cherche à atteindre des objets. Les pré-requis moteurs semblent se mettre en place, ce qui lui permet d'esquisser des mouvements de manière intentionnelle et plus contrôlée. Elle n'utilise cependant pas encore son index et ne replie pas les autres doigts de la main quand elle tend le bras. A dix mois, Madeleine se tient debout. Elle montre à ses interlocuteurs adultes des objets qu'elle tient à la main. Son contrôle de la motricité fine s'affirme et elle utilise la pince pouce-index avec dextérité. A onze mois, les premières esquisses de pointages apparaissent alors que Madeleine marche maintenant très bien à quatre pattes. Elle pointe le lieu vers lequel elle se dirige. A un an, alors que Madeleine n'a pas encore de " mots » très clairs, on note une forte augmentation du nombre de pointages. Il est rare qu'elle ne vocalise pas, ou ne babille pas en même temps. Sa mère prend en compte ces pointages dans l'interaction, y répond, et utilise également le pointage pour solliciter son attention (on note 21 pointages de la mère, dont deux en l'air pour désigner des objets absents). A partir de ce moment-là, les pointages font partie du système communicatif mis en place entre Madeleine et les adultes. Nous avons donc cherché à différencier plusieurs fonctions du pointage chez Madeleine afin de les rattacher aux fonctions des premiers mots ou des premiers énoncés. 


\section{Charlotte}

La première caractéristique de la communication visuo-gestuelle de Charlotte est l'intensité de son regard. Dès le premier enregistrement, à 7 mois, elle suit les adultes du regard avec beaucoup d'attention et de concentration. Par ailleurs, elle agite les bras en écho aux gestes de sa mère. On assiste à des premiers gestes qui semblent servir à attirer l'attention de l'autre dès l'âge de 8 mois. Ils ont été considérés comme des pointages dont la configuration n'était pas encore maîtrisée. Charlotte tend le bras vers un objet sans ouvrir les doigts dans un premier temps, puis à 9 mois, elle tend le bras avec la main ouverte, ou parfois garde seulement trois doigts tendus, relâchant le petit doigt et l'annulaire. Enfin à 10 mois, elle replie tous les doigts sauf l'index et la configuration du pointage est en place.

Les premiers pointages « précis » apparaissent donc à 10 mois, un peu plus tôt que chez Madeleine et sont beaucoup plus nombreux. Par ailleurs, dès 10 mois elle semble utiliser le pointage vers soi, vers l'interlocuteur ou vers une personne en dehors de la sphère interlocutive. Il sera donc important de vérifier par la suite, dans un travail sur l'entrée dans la grammaire, la validité des différentes thèses sur la continuité ou la discontinuité entre le geste de pointage et le signe linguistique, notamment les pronoms personnels dans le corpus de Charlotte (Morgenstern, 1997). On observe que les premiers pointages à 10 mois sont souvent accompagnés d'un autre geste ou mouvement, ce qu'on ne retrouve pas dans le corpus de l'enfant entendante. Ainsi, lorsque Charlotte veut obtenir quelque chose, le pointage peut être accompagné d'un mouvement des jambes, ou d'un mouvement du buste ou de la tête vers l'avant en direction de l'objet, ou encore le pointage vers l'objet sera suivi d'un pointage sur elle-même. Au même âge, le pointage peut être combiné à un autre geste pour «raconter» quelque chose : Charlotte s'est cogné la tête contre sa chaise haute. Quelques minutes plus tard, elle pointe sa tempe puis frappe sa tête du plat de la main. On pourrait considérer qu'il s'agit d'une combinaison de proto-signes pour raconter qu'elle s'est cogné la tête.

\subsection{Le rôle du regard}

Le nombre de pointages par séance d'une heure n'est pas une donnée suffisamment pertinente pour l'analyse des éventuelles différences formelles entre types de pointage. Nous avons alors établi la mesure du « visual checking 》 (terme utilisé en anglais pour désigner le regard sur l'autre servant à vérifier l'attention partagée) chez les deux enfants, ce que les graphiques suivants 
distinguent sous les deux catégories «regard alterné» (quand l'enfant regarde successivement l'objet pointé et l'adulte) et «regard adulte» (quand l'enfant regarde uniquement l'adulte tout en pointant une autre cible). Comme le montre le graphique 2 , les regards sur la cible du pointage («regard objet» en gris) sont prédominants chez Madeleine.

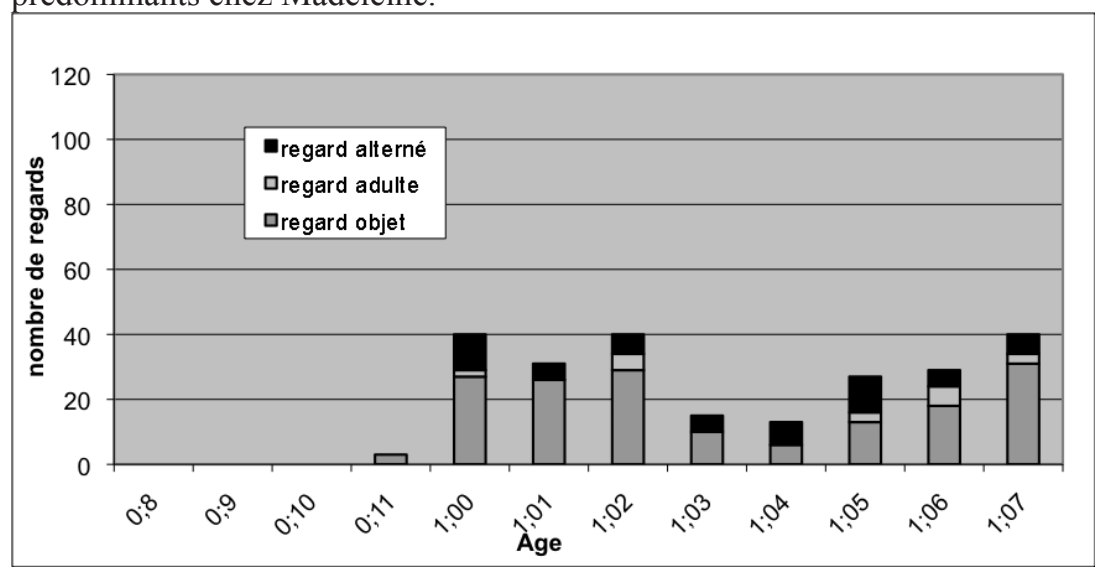

\section{Graphique 2 : Orientation des regards accompagnant les pointages de Madeleine}

Il faut cependant prendre en compte le fait que l'enfant est filmée chez elle et, pour ce qui concerne le pointage dialogique, on peut considérer que l'attention des parents lui est peut-être implicitement acquise. Elle ne ressentirait donc pas le besoin de la vérifier. Ce serait différent en situation expérimentale, dans un lieu non familier et dans des situations particulières. C'est peut-être la raison pour laquelle nos données diffèrent de celles recueillies en situation non spontanée (Butterworth 1991, 2003; Franco \& Butterworth 1996).

Par contre, dans le corpus de Charlotte, le regard semble avoir une fonction plus riche. Le graphique 3 présente l'orientation des regards accompagnant les pointages de Charlotte. 


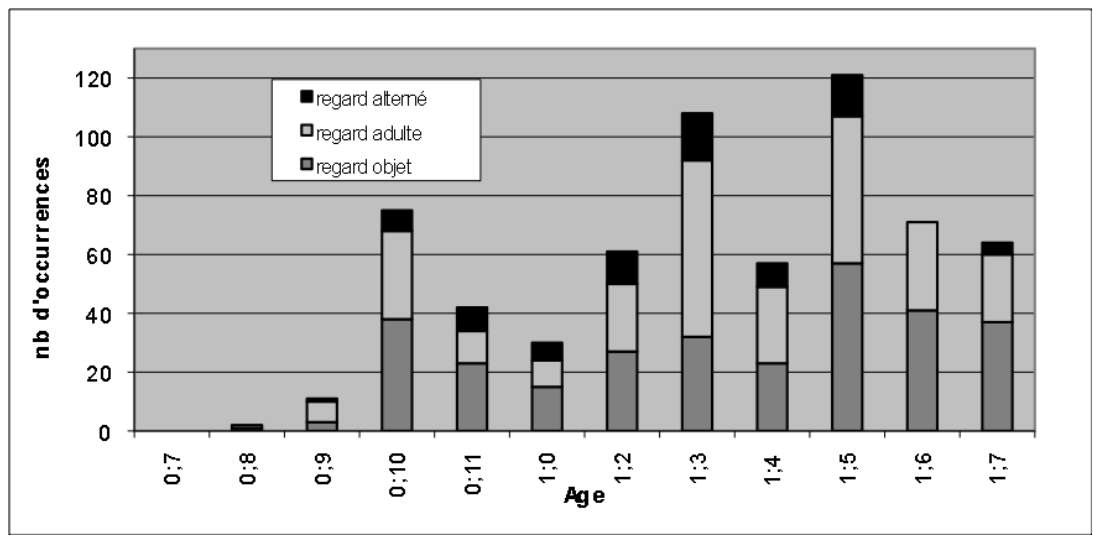

Graphique 3 : Orientation des regards accompagnant les pointages dans le corpus de Charlotte

Nous pouvons constater (en additionnant tout ce qui n'est pas en gris) que Charlotte regarde l'adulte (exclusivement ou alternativement) de façon plus précoce et beaucoup plus fréquemment que Madeleine. Par ailleurs, elle semble utiliser très tôt le regard alterné (dès 9 mois) et ses yeux sont capables de parcourir de façon maîtrisée l'espace entre l'adulte et l'objet dans les deux sens, ce que Madeleine ne fait qu'à partir de 12 mois.

Ces résultats montrent une première différence entre les paramètres qui entrent en jeu pour accompagner les pointages selon la modalité. Madeleine utilise peu le regard pour vérifier l'attention partagée, contrairement à Charlotte, petite enfant sourde pour laquelle les yeux, rappelons-le sont des « oreilles » qui « écoutent » en regardant l'autre. Cette importance du regard sur l'adulte semble devoir être mise en relation avec la position des interlocuteurs lors de l'événement-pointage. En effet, $82 \%$ des pointages de Charlotte sont produits alors qu'elle se trouve face à l'adulte, contre seulement $44 \%$ en moyenne pour Madeleine. La situation de face à face semble privilégiée dans la dyade sourde et conditionne vraisemblablement l'orientation du regard. Madeleine utilise peu le regard, mais a-t-elle cependant un autre moyen de rechercher l'attention des parents ou de s'en assurer pour compléter le geste de pointage ?

En réponse à cette question, nous avons analysé un autre paramètre important : les vocalisations et premières productions verbales. 


\subsection{Les productions vocales}

Le graphique 4 ci-dessous présente le nombre d'occurrences de pointages avec ou sans production vocale chez Madeleine.

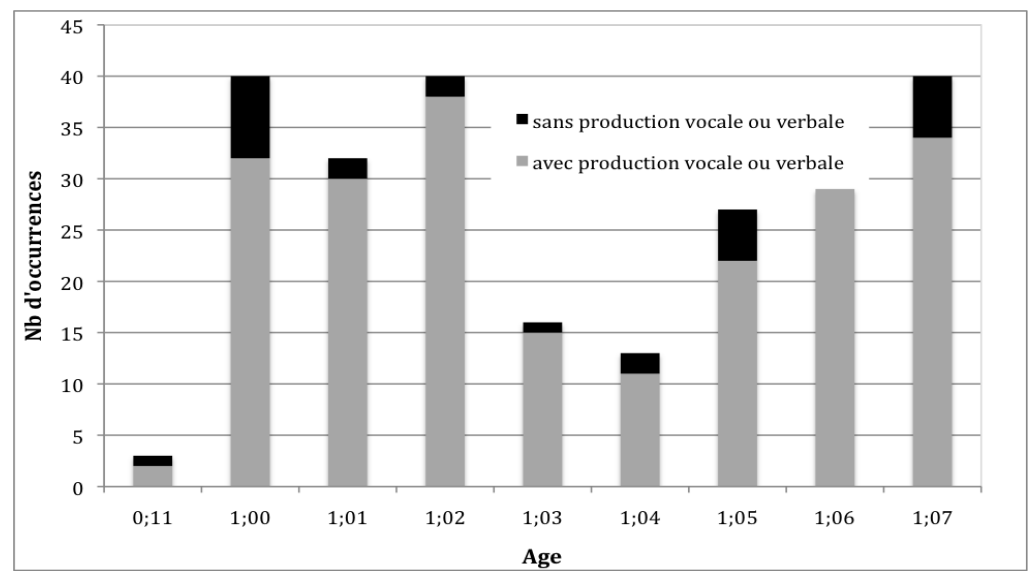

\section{Graphique 4 : Pointages de Madeleine avec et sans vocalisation}

Le graphique 4 montre la proportion importante de vocalisations et de verbalisations accompagnant les gestes de pointage. Il est rare que la petite fille pointe silencieusement. Il semblerait donc qu'elle complète le pointage, qui fait appel à la modalité visuelle, par la modalité vocale et occupe ainsi le plan sonore pour attirer l'attention de l'adulte. Une analyse fine du type de vocalisations peut permettre de regarder si les deux modalités se répètent ou se complètent. Nous avons relevé plusieurs types de vocalisations et verbalisations utilisées avec le pointage (la plupart du temps en totale simultanéité) :

1) Vocalisations peu distinctes qui sont de deux types : gémissements / cris aigus.

2) Demandes avec [mmm :], monstration avec [a :].

3) Productions verbales plus distinctes : démonstratifs [sa], dénominations [wawa], localisations [lela], commentaires sur un thème (l'enfant pointe un collier et dit « maman »; il s'agit des «combinaisons supplémentaires» décrites par Goldin-Meadow \& Morford 1994).

Les vocalisations produites par Charlotte au moment de ses pointages n'ont pas beaucoup d'impact sur l'interprétation que peuvent en faire ses 
interlocuteurs sourds. Elle produit cependant des vocalisations en général quand un affect fort est associé à son pointage et qu'elle lui donne beaucoup d'intensité (ce qui est également marqué par des mouvements du buste et de la tête ou une agitation des jambes), mais de manière beaucoup moins fréquente.

La prise en compte du regard et des productions vocales montre une disparité entre les deux enfants qui semble inhérente à la modalité de communication utilisée. Nous poursuivons donc l'étude par l'analyse des spécificités de chacune.

\section{Spécificités des pointages chez les deux enfants.}

\subsection{Les pointages de Madeleine}

Notre base de données nous a permis de corréler deux à deux tous les paramètres que nous avions codés et de distinguer deux phases dans ces corrélations.

Phase 1 : de 11 mois à 1 an et 2 mois.

Dans un premier temps, et jusqu'à 1;02 inclus, on ne trouve aucune corrélation entre les différents paramètres, sauf entre localisation de l'objet et interprétation (couplée à la réaction) de l'adulte. Il faut d'abord remarquer qu'une part importante des pointages $(51,5 \%$ en moyenne de $1 ; 00$ à $1 ; 02)$ n'entraîne pas de réaction ou de réponse de la part de l'adulte. Cependant, quand l'adulte manifeste par ses actes ou ses productions verbales qu'elle interprète le pointage, deux cas de figure sont possibles:

Si l'objet est hors de portée de l'enfant, l'adulte interprète le pointage comme une demande (proto-impératif). Il s'agit souvent de nourriture. $64 \%$ des pointages d'objets hors de portée de l'enfant sont interprétés comme impératifs.

Si l'objet est à la portée de l'enfant, l'adulte interprète le pointage comme un commentaire (proto-déclaratif). $86 \%$ des pointages d'objets à portée de l'enfant sont interprétés comme déclaratifs.

Cela signifierait qu'aucun trait distinctif émanant des gestes, du regard ou des productions vocales de l'enfant lui-même ne nous permet à ces âges de différencier les pointages concernant des demandes ou des commentaires. 


\section{Phase 2 : de 1 an 3 mois à 1 an 7 mois}

Entre 1;03 et 1;07, trois paramètres semblent corrélés deux à deux :

1) Tout comme dans la phase 1 , la situation de l'objet pointé et l'interprétation que fait l'adulte du pointage (déclaratif/impératif) sont largement corrélées. (82 à 100\% des pointages d'objets à portée de l'enfant sont interprétés comme déclaratifs ; 66 à $86 \%$ - selon l'âge - des pointages d'objets hors de portée de l'enfant sont interprétés comme impératifs.)

Ainsi, la différence de fonction entre pointage impératif et déclaratif ne viendrait pas des productions de l'enfant lui-même (association du pointage, des productions vocales et du regard) mais de paramètres externes interprétés par l'adulte. Rien dans les paramètres « internes » des productions de l'enfant ne permet de discriminer de façon déterminante les deux fonctions. Dans les exemples suivants, $\mathrm{M}$ désigne la mère. L'enfant est appelé par son prénom.

Exemple 1 (âge 1;06)

50:40. Madeleine pointe vers la cheminée sur laquelle sont posés les chocolats (elle en a mangé un quelques minutes plus tôt). Elle regarde l'adulte (caméra) en disant [dada dabala]. Sa mère arrive et dit «ah bah non ça suffit, on va fermer» et elle ferme la boîte de chocolats. L'objet est hors de portée et peut se manger. Le geste et la production de l'enfant sont interprétés comme une demande.

Exemple 2 (âge 1;06)

46:35. Madeleine pointe le pied de l'adulte et touche sa chaussure du bout du doigt en disant [asosy]. L'adulte reprend «les chaussures».

L'objet est à portée et la production vocale associée au geste est interprétée comme une dénomination. Il s'agit donc d'un « événement-pointage » que l'on peut coder comme déclaratif.

2) Le regard est corrélé à la situation de l'objet.

Si l'objet est à sa portée, l'enfant a tendance à ne regarder que l'objet (78,5 à 100\% des pointages sur un objet à portée s'accompagnent d'un regard sur l'objet), mais si l'objet est hors de portée et que l'enfant a besoin de l'attention de l'adulte ou de son aide, il va avoir tendance à regarder l'adulte (ou l'adulte et l'objet alternativement, 66 à 71,5\% des pointages sur un objet hors de portée s'accompagnent d'un regard sur l'adulte). Il nous faudra dans un deuxième temps observer plus précisément les occurrences pour tenter de déterminer si le regard précède ou suit le pointage, et donc pour établir si l'enfant vérifie l'attention conjointe avant de produire son pointage, ou s'il ne regarde l'adulte que pour vérifier l'impact de son pointage après coup. 
On pourrait faire l'hypothèse que le regard sur l'objet serait une forme non marquée (ce qui supposerait que l'enfant considère l'attention de l'adulte comme acquise par défaut lorsque l'objet se trouve à sa portée), alors que le regard sur l'adulte serait la forme marquée, nécessaire dans certaines situations pour s'assurer de son attention.

Souvent, lorsque l'enfant regarde l'adulte, la cible du pointage est nouvelle et/ou l'adulte peut se trouver dans une position qui nécessite de s'assurer de son attention (ni en face à face, ni en côte à côte).

Exemple 3 (âge 1;05)

35:34. Madeleine pointe vers une étagère en gémissant puis regarde vers sa mère, puis pointe de nouveau vers l'étagère en disant [lo]. Sa mère la prend alors dans ses bras pour la rapprocher de l'étagère sur laquelle sont posés des verres.

L'objet est hors de portée et Madeleine a besoin de sa mère, on observe que le regard est alterné entre objet et adulte.

Exemple 4 (âge 1;06)

23:43. Madeleine est en train de feuilleter le livre du personnage Poulpo. Sa mère se trouve à ses côtés. Madeleine pointe l'image en disant [pupo], tout en regardant le livre. L'objet se trouve à portée, manipulé par l'enfant et le regard reste posé sur l'objet et non sur l'adulte.

3) L'intonation est corrélée à l'attention de l'adulte

L'intonation montante semble jouer un rôle dans la mise en place de l'attention dès 1;03 (contour intonatif marqué). Par contre, lorsque l'attention est déjà établie, l'intonation est plutôt descendante (contour intonatif non marqué) ${ }^{5}$. On retrouve cette valeur chez l'adulte, la montée mélodique constituant, selon Morel et Danon-Boileau (1998) une « deixis vocale » permettant d'attirer l'attention de l'interlocuteur sur un fragment du discours.

Exemple 5 (âge 1;05)

00:35:34 Madeleine se dirige vers une autre pièce de la maison et pointe vers la chaîne hifi en regardant vers sa mère (qui vient d'arriver dans la pièce) et en disant [bali, sisis] avec une intonation montante, puis elle pointe les disques. La mère interprète cela comme une demande d'action (mettre de la musique).

Exemple $6(1 ; 06)$

57:30 Madeleine pointe vers la cheminée sur laquelle se trouve une boîte de chocolats en disant [dada, dada] avec une intonation montante et en regardant

5. Pour une analyse détaillée, voir Leroy (2001) et Leroy, Mathiot, Morgenstern (2009). 
l'observateur. Madeleine essaie donc d'attirer l'attention et de faire une demande.

Nous avons cependant un tableau un peu plus compliqué en ce qui concerne cette corrélation entre intonation et attention. D'une part, lorsque l'intonation est montante, souvent l'attention n'est pas acquise (53 à 100\% des pointages avec intonation montante sont produits lorsque l'attention n'est pas acquise) mais lorsqu'elle est descendante, l'attention peut être acquise ou non (de l'ordre de $50 \%$ pour attention acquise et $50 \%$ pour attention non acquise). Par conséquent, l'intonation serait un moyen parmi d'autres d'attirer l'attention, mais ne serait pas le seul.

D'autre part, l'intonation peut être montante même si l'attention est déjà acquise, ce qui signifierait qu'elle ne servirait pas seulement à attirer l'attention, elle peut marquer la surprise, ou, plus tard, l'opposition entre thème (ce dont il est question et qui est connu) et rhème (ce qui en est dit et qui est donc un apport d'information) (Troubetzkoy 1949), comme l'illustrent les deux exemples suivants:

Exemple 7 (âge 1;07)

49:55 Madeleine est en train de regarder Petit Ours Brun sur l'ordinateur de sa mère. Lorsque le personnage apparaît, elle pointe l'écran en disant [sa] avec intonation montante, puis elle regarde l'adulte.

Il pourrait s'agir de l'expression et du partage (confirmé par le regard) de sa surprise.

Exemple 8 (âge 1;07)

06:21. Madeleine cherche les clés de sa maison en plastique.

Adulte - elles sont pas dans la maison les clés ?

Madeleine pointe vers la maison puis vers la porte de la maison en disant [etakle intonation montante pot pala intonation descendante].

Madeleine emploie peut-être " l'événement pointage " pour répondre à une réponse à l'adulte et l'énoncé aurait alors une fonction proto-déclarative. Mais surtout, ici le thème est produit avec une intonation montante et le rhème avec une intonation descendante.

Aucun paramètre à lui seul (prosodie des productions vocales, regard, nature de l'objet, attention de l'adulte...) ne semble permettre de différencier les fonctions que prendraient les pointages de Madeleine dans l'interaction avec ses interlocuteurs. Cela pourrait indiquer que la distinction entre la fonction proto-impérative et la fonction proto-déclarative reste théorique et n'est 
pas formellement instanciée dans l'observation en milieu naturel. Cela tend à montrer que l'adulte s'appuie essentiellement sur le contexte pour interpréter les pointages de l'enfant. La localisation de l'objet dans l'espace, le fait qu'il soit atteignable ou pas pour l'enfant, la nature de l'objet permettraient à l'adulte d'interpréter si l'enfant fait une demande ou un commentaire. Ainsi si l'enfant pointe des chocolats qu'elle ne peut pas attraper, l'adulte peut penser qu'elle les lui demande. Si l'enfant pointe le dessin sur sa robe, l'adulte pensera alors qu'elle fait un commentaire, tout comme pour le pointage vers un avion qui passe dans le ciel.

On peut cependant considérer, à partir des premiers résultats de nos corrélations de paramètres, que le paramètre objet nouveau ou qui n'est pas sous l'attention conjointe « demande » à être marqué. Il le serait soit par l'intonation (montante), soit par le regard (on considère donc le regard sur l'adulte comme un regard marqué). Dans ce cas là, l'objectif de l'enfant est d'attirer ou vérifier l'attention de l'adulte, que ce soit par le regard ou par la voix en complément du geste. Le regard permettrait à l'enfant de vérifier l'attention de l'adulte, la voix d'attirer cette attention et le geste de la diriger vers la cible choisie par l'enfant.

Pour résumer ce que nous avons constaté sur la période observée chez l'enfant entendante:

- Dans un premier temps, nous ne trouvons pas de corrélation de paramètres qui nous permettrait de différencier les "événements pointages 》 (associés au regard et aux productions vocales) en terme de fonction pragmatique.

- Dans un deuxième temps, la prosodie que nous avons appelée marquée (montante) semble liée à la volonté d'attirer l'attention de l'adulte ou d'exprimer la surprise. Le regard sur l'adulte, que nous avons qualifié de marqué est un moyen de vérifier que son attention est bien portée sur un objet de " discours » commun. Il n'est cependant pas évident pour Madeleine de combiner le pointage gestuel dans la direction de l'objet, le regard sur l'autre dans une autre direction et d'exercer également éventuellement une certaine maitrise de sa prosodie avec une intentionnalité, des fonctions communicatives. Madeleine semble se concentrer sur deux paramètres à la fois et les combiner afin d'obtenir l'attention de l'adulte.

Dans la suite de notre corpus, à partir de 1;07, les productions vocales et surtout verbales vont se diversifier. On verra apparaître des contours intonatifs plats, mais également des contours en cloche avec une distinction entre la reprise d'un thème (contour montant) et son association avec un rhème (contour descendant) tout comme dans la langue adulte. 
Par ailleurs, Madeleine va mettre en place une utilisation beaucoup plus fine du regard sur l'autre, du regard alterné entre la cible du pointage et l'adulte et semble pouvoir maitriser à la fois le mouvement et la direction de son geste de pointage, l'intonation de ses productions verbales et la direction de son regard.

Chacun de ces paramètres semble donc avoir un rôle spécifique :

- les vocalisations permettent d'attirer l'attention de l'interlocuteur,

- le geste de pointage permet d'orienter l'attention de l'interlocuteur dans l'espace,

- le regard permet de vérifier que l'attention de l'adulte est bien portée sur l'objet de discours désigné.

Or si Madeleine, petite fille entendante, peut combiner ces trois éléments et maîtriser progressivement leur utilisation, qu'en sera-t-il de Charlotte, petite fille sourde, entourée d'adulte sourds, qui n'a pas à sa disposition les vocalisations ou la finesse de leur prosodie pour accompagner ses gestes de pointage ? Afin de mettre en regard les « événements » pointages des enfants entendants et des enfants sourds-signeurs, nous avons donc commencé ${ }^{\text {à }}$ analyser les pointages et les éléments qui lui sont associés dans le corpus de Charlotte.

\subsection{Les pointages de Charlotte}

Nous n'avons pas encore analysé et quantifié de façon très fine les corrélations de paramètres dans les évènements-pointage de Charlotte. Nous pouvons cependant faire les observations générales suivantes.

\section{1) Le rôle des productions vocales}

Avant d'écarter totalement le rôle des productions vocales dans les « évènements-pointage » chez Charlotte, nous avons analysé une à une les occurrences de pointage et cherché à voir si elles s'accompagnaient de vocalisations. Ces dernières sont en fait assez rares et semblent accompagner les pointages dans un contexte où Charlotte exprime un affect, la surprise, la joie, la colère notamment, et intensifie ainsi l'ensemble de sa production. Ces vocalisations, rares, sont cependant plus fréquentes en début de corpus. La voix accompagne l'expression de l'affect et la dépense d'énergie. En milieu de corpus, ses émissions vocales tendent à disparaître. Il semblerait que cela corresponde à une plus grande maîtrise de son corps. De manière intéressante, ces observations soulignent

6. Il s'agit ici de présenter nos tout premiers résultats. 
donc l'utilisation intentionnelle des productions vocales chez Madeleine et le rôle grandissant et contrôlé de l'intonation et inversement leur diminution chez Charlotte en tant que signaux qui n'ont pas de valeur communicationnelle.

\section{2) Le rôle du regard}

Une différence essentielle dans les interactions au sein des dyades mère-Charlotte et mère-Madeleine réside dans le regard que porte la mère de Charlotte sur elle : ses yeux constituent le lien essentiel avec sa fille et lui permettent également de s'assurer de son bien-être et de sa sécurité. Son champ visuel est ainsi, de par sa pratique, plus large que celui d'un adulte entendant. La plupart du temps, son attention visuelle est dirigée, au moins partiellement, sur Charlotte. Cependant, quand pour une raison quelconque, le regard de sa mère n'est pas porté sur elle et qu'elle veut attirer son attention, Charlotte accompagne son pointage de mouvements assez intenses du corps (buste, tête, jambes). Elle peut également répéter son pointage. Une autre stratégie, très étonnante pour les entendants, est l'absence totale de mouvement accompagné d'un regard fixe sur sa mère lorsque Charlotte cherche à attirer son attention. Ainsi l'augmentation (agitation) ou la disparition (immobilisme) du geste et des mouvements qui l'accompagnent seraient des formes marquées par rapport à un geste « normal».

L'analyse quantitative des différents paramètres codés grâce au logiciel ELAN ne fait pas apparaitre de corrélation entre l'orientation du regard et la fonction du pointage (impérative ou déclarative), ni entre l'orientation du regard et la place de l'objet dans le discours (nouveau ou déjà mentionné). Par contre, tout comme chez Madeleine, on peut observer chez Charlotte une corrélation importante entre l'orientation du regard et la situation de l'objet : lorsque l'enfant regarde l'adulte, l'objet est généralement hors de sa portée (dans $86 \%$ des cas pour Madeleine et $88 \%$ pour Charlotte). Au contraire, lorsque l'enfant regarde l'objet, ce dernier est le plus souvent à sa portée.

\section{3) La situation de l'objet}

Nous avons pu noter que la situation de l'objet (à portée ou hors de portée de l'enfant) semblait constituer l'un des critères les plus robustes pour l'interprétation du pointage par l'adulte (pointage de commentaire ou de demande) dans le corpus de Madeleine. L'analyse du corpus de Charlotte fait apparaître le même type de corrélation, entre la situation de l'objet et la fonction du pointage, à partir de 1;03. En effet, lorsque l'objet est hors de portée de l'enfant, l'adulte 
a tendance à interpréter le pointage comme impératif (avec une moyenne de $54 \%$ des pointages d'objets hors de portée de l'enfant interprétés comme des demandes), mais de manière beaucoup moins saillante que dans le corpus de Madeleine. En effet, de nombreux pointages d'objets hors de portée sont interprétés comme déclaratifs. Ce pourcentage chute à l'âge de 1;07, ce qui peut être expliqué par l'augmentation des signes combinés aux pointages, facilitant de ce fait leur interprétation.

Lorsque l'objet est à portée de l'enfant, l'adulte interprète le pointage comme déclaratif dans 69 à $90 \%$ des cas. Ce pourcentage, même s'il est relativement important, reste moins élevé que dans le corpus de Madeleine. Nous pouvons peut-être en déduire que le paramètre « situation de l'objet » serait l'un des critères permettant à l'interlocuteur adulte de faire la différenciation entre d'éventuelles fonctions de l'événement-pointage, mais qu'il ne serait pas le seul, ce que la suite de ce travail permettra peut-être de démontrer.

\section{4) La non-simultanéité}

$\mathrm{Si}$, chez Madeleine, le pointage et la production vocale qui va devenir une production verbale peuvent être simultanés, Charlotte enchaîne en revanche le pointage avec un signe dès 13 mois. On assiste donc à une combinaison dans la linéarité temporelle (et non dans la simultanéité) qui marquerait l'émergence d'une proto-syntaxe gestuelle dont l'élément central semble être le pointage.

\section{Conclusion}

Le pointage permet à l'enfant de segmenter le milieu environnant, de singulariser un élément saillant pour lui et d'en faire un référent. L'enfant peut alors parler de cet objet, pour lui, mais bien sûr pour l'autre, le nommer, le demander, le commenter. Son activité de monstration lui permet de faire ses premiers pas dans la prédication : il extrait un élément du réel qui l'entoure et l'insère dans une proto-structure syntaxique à deux éléments. C'est à partir de cet ancrage pragmatique que l'enfant entre pleinement dans le langage.

Notre comparaison entre les premiers pointages de Charlotte, une enfant sourde-signeuse élevée dans un entourage pratiquant la LSF, et Madeleine une enfant entendante élevée dans une famille parlant le français n'est encore qu'une esquisse. Mais nous avons pu déjà mettre au jour l'importance de la combinaison entre différents paramètres et la nécessité d'effectuer des corrélations pour analyser «l'événement-pointage». 
Chez Madeleine, lors de ces « événements-pointages », la voix sert à attirer l'attention mais aussi plus tard à donner un contenu référentiel et informationnel, le geste à localiser l'objet d'attention pour l'autre et le regard (vers la fin de notre corpus) à vérifier que l'attention est portée sur l'objet de « discours ». Chez Charlotte, l'enchaînement d'un pointage avec un proto-signe - c'est-à-dire la combinaison de deux manifestations manuo-gestuelles - apparaît relativement tôt et le regard semble avoir une qualité plus fine et des fonctions plus diversifiées qu'il s'agit d'étudier en détail en fonction des autres paramètres que nous avons analysés dans le corpus de Madeleine.

Dans les deux dyades, il ne semble donc pas y avoir de trait distinctif qui à lui seul permettrait de discriminer des fonctions sémantico-pragmatiques au niveau de la production : l'interlocuteur adulte semble s'appuyer en premier lieu dans les deux cas sur la nature et la position de l'objet pointé pour interpréter les productions des événements-pointages (geste + regard + éventuelle production vocale/production de mots ou de signes) en contexte. Cette étude, qui porte sur les moments d'interaction comportant des pointages, nous rappelle que tout acte communicationnel est à interpréter en situation, dans son contexte dialogique et en prenant en compte la dimension multi-modale du langage.

\section{Références}

Bates, E. (1976). Language and context : the acquisition of pragmatics. New York: Academic Press.

Bates, E., Benigni, L., Bretherton, I., Camaioni, L. \& Volterra, V. (1979). The emergence of symbols: Cognition and communication in infancy. New York: Academic Press.

Bates, E., Camaioni, L. \& Volterra, V. (1975). The acquisition of performatives prior to speech. Merrill-Palmer Quarterly $\mathrm{n}^{\circ}$ 21, 205-226.

Bonvillian, J.D., Orlansky, M.D. \& Folden, R.J. (1994). Early sign language acquisition: implications for theories of language acquisition. In V. Volterra \& C.J. Erting (eds.), From Gesture to Language in Hearing and Deaf Children, 219-232. Washington, DC: Gallaudet University Press.

Boyes Braem, P. (1994). Acquisition of the handshape in American Sign Language: A preliminary analysis. In V. Volterra \& C.J. Erting, (eds.), From Gesture to Language in Hearing and Deaf children, 107-127. Washington, DC: Gallaudet University Press.

Brigaudiot, M. \& Danon-Boileau, L. (2002). La naissance du langage dans les deux premières années. Paris: P.U.F.

Bruner, J.S. (1977). Early social interaction and language acquisition. In H.R. Schaffer (ed.), Studies in Mother-Child Interaction, 271-289. London: Academic Press. 
Bruner, J.S. (1983). Child's talk : learning to use language. New York: Norton.

Butterworth, G. (1991). The ontogeny and philogeny of joint visual attention. In A. Whiten (ed.), Natural Theories of Mind, 223-232. Oxford: Basil Blackwell.

Butterworth, G. (2003). Pointing is the royal road to language for babies. In S. Kita (ed.), Pointing: Where Language, Culture, and Cognition Meet, 9-34. Mahwah, NJ: Lawrence Erlbaum.

Cabrejo-Parra, E. (1992). Deixis et opérations symboliques. In L. Danon-Boileau \& M.-A. Morel (eds.), La Deixis, 409-414. Paris: P.U.F.

Capirci, O., Iverson, J.M., Pizzuto, E. \& Volterra, V. (1996). Gestures and words during the transition to two-word speech. Journal of Child Language $\mathrm{n}^{\circ} 23,645-673$.

Caselli, M.C. \& Volterra, V. (1994). From communication to language in hearing and deaf children. In V. Volterra \& C.J. Erting (eds.), From Gesture to Language in Hearing and Deaf Children, 263-277. Washington, DC: Gallaudet University Press.

Conlin, K. E., Mirus, G. R., Mauk, C. \& Meier, R. (2000). The acquisition of first signs: place, handshape, and movement. In C. Chamberlain, J. P. Morford \& R.I. Mayberry (eds.), Language Acquisition by Eye, 51-69. Mahwah, NJ: Lawrence Erlbaum.

Emmorey, K. (2002). Language, cognition and the brain: Insights from sign language research. Mahwah, NJ: Lawrence Erlbaum.

Engberg-Petersen, E. (1993a). Space in Danish Sign Language. Hamburg: Signum Press.

Engberg-Pedersen, E. (1993b). The ambiguous point. Sign Post $\mathrm{n}^{\circ}$ 6/2, 2-8.

Folven, R.J., Bonvillian, J.D. \& Orlansky, M.D. (1984/1985). Communicative gestures and early sign language acquisition. First Language $\mathrm{n}^{\circ}$ 5, 129-144.

Franco, F., \& Butterworth, G. (1996). Pointing and social awareness : declaring and requesting in the second year. Journal of Child Language $\mathrm{n}^{\circ} 23,307-337$.

Goldin-Meadow, S. \& Morford, M. (1994). Gesture in early child language. In V. Volterra \& C.J. Erting (eds.), From Gesture to Language in Hearing and Deaf Children, 249-262. Washington, DC: Gallaudet University Press.

Goldin-Meadow, S., \& Butcher, C. (2003). Pointing toward two-word speech in young children. In S. Kita (ed.), Pointing : Where Language, Culture and Cognition Meet, 85-107. Mahwah, NJ: Lawrence Erlbaum.

Guidetti, M. (1998). Les usages des gestes conventionnels chez les enfants. In J. Bernicot, H. Marcos, C. Day, M. Guidetti, V. Laval, J. Rabain-Jamin \& G. Babelot (eds.), De l'Usage des Gestes et des Mots chez l'Enfant, 27-50. Paris: Armand Colin.

Guidetti, M. (2003). Pragmatique et psychologie du développement: comment communiquent les jeunes enfants. Paris: Belin.

Harris, M., Barlow-Brown, F. \& Chasin, J. (1995). The emergence of referential understanding: pointing and the comprehension of object names. First Language $\mathrm{n}^{\circ} 15,19-34$. 
Kaye, K. (1982). The mental and social life of babies. Chicago: Chicago University Press.

Leung, E. H. \& Rheingold, H. L. (1981). Development of pointing as a social gesture. Developmental Psychology $\mathrm{n}^{\circ}$ 17, 215-220.

Leroy, M. (2001). Mise en place et développement de l'intonation et de la mimo-gestualité chez l'enfant entre 0;2 et 1;10. Thèse de doctorat, Université de Paris 3-Sorbonne Nouvelle.

Leroy, M., Mathiot, E., Morgenstern, A. (2009). Pointing gestures, vocalizations and gaze: two case studies. In J. Zlatev, M. Andrén, M. Johansson Falck \& C. Lundmark (eds.), Studies in Language and Cognition, 402-420. Newcastle upon Tyne: Cambridge Scholars Publishing.

Liszkowski, U., Carpenter, M., Striano, T. \& Tomasello, M. (2006). Twelve- and 18months-olds point to provide information for others. Journal of Cognition and Development $\mathrm{n}^{\circ}$ 7, 173-187.

Lock, A., Young, A., Service, V. \& Chandler, P. (1994). Some observations on the origins of the pointing gesture. In V. Volterra \& C.J. Erting (eds.), From Gesture to Language in Hearing and Deaf Children, 42-55. Washington, DC: Gallaudet University Press.

MacWhinney, B. (2000). The CHILDES project: Tools for analyzing talk. Mahwah, NJ: Lawrence Erlbaum.

Marcos, H. (1998). De la communication prélinguistique au langage: formes et fonctions. Paris: L'Harmattan.

Masataka, N. (2003). From index-finger extension to index-finger pointing: ontogenesis of pointing in preverbal infants. In S. Kita (ed.), Pointing: Where Language, Culture, and Cognition Meet, 69-84. Mahwah, NJ: Lawrence Erbaum.

Morel, M.-A. \& Danon-Boileau, L. (1998). Grammaire de l'intonation, l'exemple du français, Paris : Ophrys.

Morgan, G. (2009). What can deaf children's sign language tell us about language and communication development, Conférence présentée au Child Language Seminar, 12 janvier 2009, Maison de la Recherche, Paris.

Morgenstern, A. (1997). L'enfant sourd énonciateur signeur: 1'auto-désignation chez l'enfant en Langue des Signes Française. Lidil n 15, 119-141.

Moore, C. \& Dunham, P. (eds.) (1995). Joint attention: its origins and role in development. Hillsdale, NJ : Erlbaum.

Morford, M. \& Goldin-Meadow, S. (1992). Comprehension and production of gesture in combination with speech in one-word speakers. Journal of Child Language $\mathrm{n}^{\circ} 19,559-580$.

Murphy, C.M. \& Messer, D.J. (1977). Mothers, infants and pointing: a study of a gesture. In H.R. Schaffer (ed.), Studies in Mother-Infant Interaction, 325-354. London: Academic Press.

Ninio, A. \& Bruner, J.S. (1978). The achievement and antecedents of labeling. Journal of Child Language $\mathrm{n}^{\circ}$ 5, 1-15. 
Pika, S., Liebal, K., Call, J., \& Tomasello, M. (2005). The gestural communication of apes. Gesture $\mathrm{n}^{\circ} 5,1 / 2,41-56$.

Schaffer, H.R. (1977). Early interactive development. In H.R. Schaffer (ed.), Studies in Mother-Child Interaction, 3-16. London : Academic Press.

Schaffer, H.R. (1984). The child's entry into the social world. London: Academic Press.

Schick, B. (2007). Le développement de la langue des signes américaine. Enfance $\mathrm{n}^{\circ}$ 59/3, 220-227.

Southgate, V., Van Maanen, C. \& Csibra, G. (2007). Communication to cooperate or communication to learn? Child Development $\mathrm{n}^{\circ} 78,735-740$.

Tomasello, M. (1999). The cultural origins of human cognition. Cambridge, M.A.: Harvard University Press.

Tomasello, M. (2003). Constructing a language: a usage-based theory of language acquisition. Cambridge, MA : Harvard University Press.

Tomasello, M. \& Camaioni, L. (1997). A comparison of the gestural communication of apes and human infants. Human Development $\mathrm{n}^{\circ}$ 40, 7-24.

Troubetzkoy, N. (1949). Principes de phonologie, Paris : Klincksieck.

Van den Bogaerde, B. (2000). Input and interaction in deaffamilies. Thèse de doctorat. Université d'Amsterdam.

Volterra, V., Caselli, M.C., Capirci, O. \& Pizzuto, E. (2005). Gesture and the emergence and development of language. In M. Tomasello \& D. Slobin (eds.), Beyond Nature-Nurture: Essays in Honor of Elizabeth Bates, 3-40. Mahwah, NJ: Lawrence Erlbaum.

Vygotsky, L. (1985). Pensée et langage. Paris: Editions Sociales.

Werner, H. \& Kaplan, B. (1963). Symbol formation. Hillsdale, NJ: Erlbaum.

Wundt, W. (1912). Völkerpsychologie: die Sprache. Leipzig: Engelmann.

\begin{abstract}
Pointing emerges thanks to the mastery of motor and cognitive prerequisites and it facilitates symbolization processes. It enables children to designate an object as a focus for joint attention thus paving the way to early language acquisition.

In this paper, we compare the data taken from two longitudinal follow-ups of a French-speaking child and a deaf child acquiring French Sign Language (LSF), aged 7 months to 1 year and 7 months, filmed at home with their parents once a month. Traditionally, researchers have attributed two functions to pointing gestures: they could be proto-imperative or proto-declarative. We closely examine each pointing gesture used by the hearing and deaf children in order to determine whether there are significant differences according to these two functions. Thanks to a multimodal approach, we will try to determine
\end{abstract}


whether the combination of different features in the pointing-event, such as gaze, the prosody of the vocal productions, the status of the object in the interaction, the focus of attention or the location of the interlocutor could be significant. 
